\title{
Duas novas espécies de Labicymbium (Araneae: Linyphiidae) do sul do Brasil
}

\author{
Everton N. L. Rodrigues
}

Programa de Pós-Graduação em Biologia Animal, Departamento de Zoologia, Instituto de Biociências, Universidade Federal do Rio Grande do Sul. Avenida Bento Gonçalves 9500, Bloco IV, Prédio 43435, 91501-970 Porto Alegre, Rio Grande do Sul, Brasil. Bolsista do CNPq. E-mail: enlrodrigues@yahoo.com.br

\begin{abstract}
Two new species of Labicymbium (Araneae: Linyphiidae) from south of Brazil. In this paper, two new species of Labicymbium Millidge, 1991 from Brazil are described: Labicymbium otti sp. nov. and Labicymbium curitiba sp. nov. Specimens of $L$. otti sp. nov. were collected from the State of Rio Grande do Sul and are characterized by the presence of an distal suprategular apophyses projected, paracymbium with round margin and radix little developed. The female of this species have copulatory ducts shorter forming a loop in posterior portion. Labicymbium curitiba sp. nov., from the State of Paraná, depicts a distal suprategular apophysis prominent and round, basal hematodochal sclerite on the subtegulum, palpal tibia long, retromedian cymbial process less pointed than those of the close species, paracymbium with projection on the subtegulum and basal hematodochal sclerite. Labicymbium rusticulum (Keyserling, 1891) is reported from new locations in the State of Rio Grande do Sul. KEY WORDS. Erigoninae; Neotropical; spiders; taxonomy.
\end{abstract}

RESUMO. Nesse trabalho duas novas espécies de Labicymbium Millidge, 1991 do Brasil são descritas: Labicymbium otti sp. nov. e Labicymbium curitiba sp. nov. Espécimes de L. otti sp. nov. foram coletados no estado do Rio Grande do Sul e são caracterizados pela presença de uma apófise suprategular distal projetada, paracímbio com margem arredondada e radix menos desenvolvido. A fêmea dessa espécie apresenta ductos copulatórios curtos formando uma alça na porção posterior. Espécimes de $L$. curitiba sp. nov., coletados no estado do Paraná, apresentam uma apófise suprategular distal proeminente e arredondada, esclerito hematodocal basal sobre o subtégulo, tíbia do palpo longa, processo cimbial retromediano menos afilado do que aqueles das espécies próximas, paracímbio com projeção sobre o subtégulo e esclerito hematodocal basal. Labicymbium rusticulum (Keyserling, 1891) é registrada de outras localidades do estado do Rio Grande do Sul.

PALAVRAS-CHAVE. Aranhas; Erigoninae; Neotropical; taxonomia.

Labicymbium foi proposto por MilLidge (1991) que designou como espécie-tipo L. sturmi Millidge, 1991 da Colômbia. Após a recente revisão das aranhas neotropicais Erigoninae, realizada por Miller (2007), esse gênero é composto por 18 espécies de aranhas. Dessas, somente L. rancho Ott \& Lise, 1997 e L. rusticulum (Keyserling, 1891) são registradas para o Brasil.

Espécies de Labicymbium caracterizam-se pela presença no palpo do macho do processo cimbial retromediano e retrobasal e esclerito hematodocal basal; as fêmeas são diagnosticadas por processos fortemente curvados que surgem das laterais do epígino, separados por uma proeminência mediana, arredondada ou subretangular (Millidge 1991, Miller 2007). Conforme Miller (2007), as espécies brasileiras fogem um pouco desse padrão. Nas fêmeas os dois processos surgem direto da margem posterior do epígino das fêmeas; no macho de L. rancho o processo cimbial retromediano está reduzido.

Nesse trabalho são descritas duas novas espécies do sul do Brasil; são apresentadas novas ilustrações do palpo do macho e epígino da fêmea de $L$. rusticulum e novos registros para a espécie.

O material descrito está depositado na coleção aracnológica do Museu de Ciências Naturais, Fundação Zoobotânica do Rio Grande do Sul (MCN, E.H. Buckup) e Museu de Ciências e Tecnologia da Pontifícia Universidade Católica do Rio Grande do Sul (MCTP, Arno A. Lise), ambos em Porto Alegre. A posição da tricobótria no metatarso da perna I (TmI) foi calculada conforme Millidge (1980). Terminologia e descrições seguem Miller (2007). O estudo das estruturas internas da genitália da fêmea foi realizado mergulhando o epígino em ácido láctico $85 \%$ ou óleo de cravo. Todas as medidas são fornecidas em milímetros. Abreviaturas utilizadas para os olhos: (OLA) olhos laterais anteriores, (OLP) olhos laterais posteriores, (OMA) olhos médios anteriores e (OMP) olhos médios posteriores.

\section{Labicymbium otti sp. nov.}

Figs $1-8$

Material-tipo. Holótipo macho, BrasiL, Rio Grande do Sul: São Francisco de Paula (Centro de Pesquisas e Conservação da Natureza Pró-Mata), 12-15.VI.1997, R. Ott leg., pitfal-trap (MCN 43581). Parátipos: fêmea, mesma localidade, data e coletor do holótipo (MCN 43582); 2 machos e 3 fêmeas, mesma localida- 

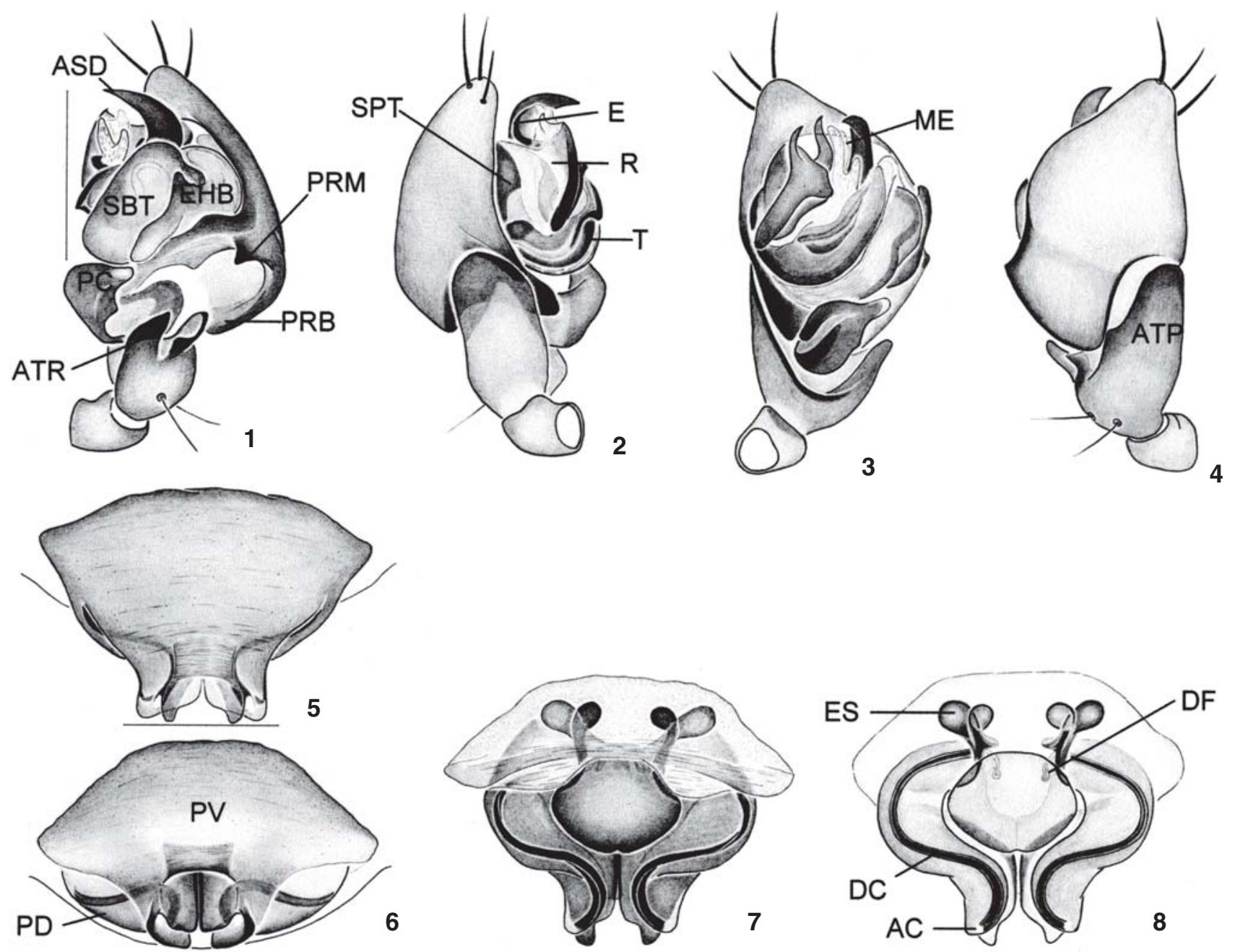

Figuras 1-8. Labicymbium otti sp. nov. (1-4) Palpo do macho: (1) vista retrolateral; (2) vista prolateral; (3) vista ventral; (4) vista dorsal; (5-8) epígino: (5) vista ventral; (6) vista posterior; (7) vista dorsal; (8) vista dorsal, clarificado. (AC) abertura de copulação, (ASD) apófise suprategular distal, (ATP) apófise tibial prolateral, (ATR) apófise tibial retrolateral, (DC) ducto de copulação, (DF) ducto de fertilização, (E) êmbolo, (EHB) esclerito hematodocal basal, (ES) espermateca, (ME) membrana embólica, (PC) paracímbio, (PD) placa dorsal, (PRM) processo cimbial retromediano, (PRB) processo cimbial retrobasal, (PV) placa ventral, (R) radix, (SBT) subtégulo, (SPT) supratégulo, (T) tégulo. Barras: 0,25 mm.

de, data e coletor do holótipo (MCN 43583-43585); 2 machos e 1 fêmea, mesma localidade e coletor do holótipo, 11.IV.1996 (MCTP 8772).

Etimologia. O nome específico é dedicado ao aracnólogo Ricardo Ott, coletor dos espécimes estudados.

Diagnose. O palpo do macho de L. otti sp. nov. assemelha-se ao de L. rusticulum (Keyserling, 1891) (Отт \& Lise 1997, figs 1-4; vide Figs 16-19) por apresentar em comum o processo cimbial retromediano afilado e apófise suprategular distal projetada, difere por esta última ser pontiaguda distalmente (Figs 14), paracímbio com margem arredondada (Fig. 1) e radix menos desenvolvido não ultrapassando o tégulo (Fig. 2). Fêmea com epígino similar ao de L. rusticulum (Keyserling, 1891) (OTт \& LisE
1997, figs 5-7; vide Fig. 20) por compartilhar dois processos que conduzem os ductos copulatórios (Figs 5 e 6), mas separam-se por serem curtos em $L$. otti e pelo percurso dos ductos de copulação formarem uma alça na porção posterior (Figs 7 e 8). Descrição. Holótipo macho. Comprimento total 1,79. Carapaça, comprimento 0,87, largura 0,72, altura 0,52. Clípeo, altura 0,15. Esterno, comprimento 0,50, largura 0,47. Abdome, comprimento 0,95 , largura 0,67 , altura 0,72 . Fórmula das pernas $1 / 2 / 4 / 3$. Comprimento $1 / 2 / 3 / 4$ : fêmures $0,80 / 0,70 / 0,55 / 0,57$; patelas $0,25 / 0,25 / 0,20 / 0,20$; tíbias $0,72 / 0,65 / 0,50 / 0,60$; metatarsos $0,62 / 0,57 / 0,50 / 0,55$; tarsos $0,44 / 0,40 / 0,35 / 0,37$. Total 2,83/2,57/2,10/2,29. Posição da tricobótria no metatarso da perna I (TmI) 0,4. Todos metatarsos com tricobótria. Carapaça

Revista Brasileira de Zoologia 25 (2): 323-327, June, 2008 
castanho-avermelhada com pontilhados negros, glabra com somente uma fila de 2-3 cerdas dorsais. Área ocular e fóvea enegrecidas. Clípeo glabro. Quelíceras castanho-avermelhadas, com processo espiniforme frontal, cinco dentes na promargem e quatro na retromargem. Lábio castanho escuro. Esterno castanho-avermelhado com bordas mais escuras. Enditos e pernas castanhas, coxas com pontilhados negros. Fila ocular anterior 0,28 e posterior 0,32 . OLA, OMP e OLP do mesmo tamanho $(0,07)$ e OMA os menores $(0,04)$. Abdome mais longo do que largo, castanho escuro, bastante piloso. Fiandeiras castanhas, com tonalidade mais clara que o ventre do abdome. Palpo com êmbolo adjacente à apófise suprategular distal (Fig. 2), com porção terminal junto à membrana embólica (Figs 2 e 3), apófise tibial retrolateral pontiaguda e esclerotinizada distalmente, esclerito hematodocal basal longo, sobre o subtégulo (Fig. 1).

Parátipo fêmea (São Francisco de Paula, MCN 43582). Comprimento total 2,00. Carapaça, comprimento 0,92, largura 0,72, altura 0,50. Clípeo, altura 0,13. Esterno, comprimento 0,50, largura 0,50 . Abdome, comprimento 1,10 , largura 0,85 , altura 0,87 . Fórmula das pernas $1 / 4 / 2 / 3$. Comprimento $1 / 2 / 3 / 4$ : fêmures $0,82 /$ $0,70 / 0,57 / 0,85$; patelas $0,25 / 0,25 / 0,25 / 0,25$; tíbias $0,67 / 0,55 / 0,50 /$ 0,72; metatarsos 0,65/0,55/0,52/0,60; tarsos 0,44/0,42/0,37/0,40. Total 2,83/2,47/2,21/2,82. Posição da tricobótria no metatarso da perna I (TmI) 0,4. Todos metatarsos com tricobótria. Carapaça castanho-avermelhada com pequenos pontilhados negros, glabra, exceto uma fila de 2-3 cerdas dorsais. Área ocular e fóvea enegrecidas. Clípeo glabro. Quelíceras castanho-avermelhadas com cinco dentes na promargem e quatro na retromargem. Esterno castanho. Enditos e pernas castanhos, coxas com pontilhado negro. Fila ocular anterior 0,30 e posterior 0,35 . OLA, OMP e OLP do mesmo tamanho $(0,07)$ e OMA os menores $(0,05)$. Abdome mais longo do que largo, castanho-escuro, piloso. Fiandeiras castanhas, com tonalidade mais clara que o ventre do abdome. Colulo bastante desenvolvido. Epígino com espermatecas ovais, ductos de fertilização enovelados, pouco esclerotinizados com origem na porção posterior das espermatecas e ductos de copulação longos (Figs 7 e 8); placa dorsal mais curta que a ventral (Figs 5 e 6).

Variação comprimento total. Machos (10 espécimes medidos): 1,77-1,82; fêmeas (10): 1,96-2,02. Variação do colorido corporal. Por vezes, cefalotórax com tonalidade mais clara, castanho-claro e abdome castanho.

Material examinado. Brasil, Rio Grande do Sul: São Francisco de Paula (Centro de Pesquisas e Conservação da Natureza PróMata), 15 machos, 5 fêmeas, 23.XI.2000-02.II.2001, R. Ott leg. pitfal-trap (MCN 39398-39400); mesma localidade e coletor, 3 machos, 10 fêmeas, 17.XI.1996-15.VI.1997 (MCN 43586, 43587).

Distribuição. Sul do Brasil (Rio Grande do Sul).

\section{Labicymbium curitiba sp. nov.} Figs 9-15

Material-tipo. Holótipo macho, BRASIL, Paraná: Curitiba, 04.XI.1993, M. Pie leg. (MCN 24493). Parátipo macho, mesma localidade e coletor do holótipo, 18.XI.1993 (MCN 24494).
Etimologia. O substantivo específico em aposição referese à localidade-tipo.

Diagnose. O palpo do macho de L. curitiba sp. nov. assemelha-se ao de L. rusticulum (Keyserling, 1891) (Отт \& Lise 1997, figs 1-4; vide Figs 16-19) pela apófise suprategular distal proeminente e arredondada e de $L$. otti por apresentar o esclerito hematodocal basal sobre o subtégulo (Fig. 12), difere pela tíbia do palpo longa (Figs 12-15), processo cimbial retromediano menos afilado, paracímbio com projeção sobre o subtégulo e esclerito hematodocal basal (Fig. 12).

Descrição. Holótipo macho. Comprimento total 2,22. Carapaça, comprimento 1,12, largura 0,87, altura 0,52. Clípeo, altura 0,20. Esterno, comprimento 0,57, largura 0,60. Abdome, comprimento 1,12 , largura 0,72 , altura 0,57 . Fórmula das pernas $1 / 4 / 2 / 3$. Comprimento $1 / 2 / 3 / 4$ : fêmures $0,97 / 0,87 / 0,70 /$ 0,92 ; patelas $0,27 / 0,25 / 0,25 / 0,27$; tíbias $0,89 / 0,80 / 0,65 / 0,92$; metatarsos $0,75 / 0,67 / 0,60 / 0,75$; tarsos $0,50 / 0,44 / 0,47 / 0,40$. Total 3,38/3,03/2,67/3,26. Posição da tricobótria no metatarso da perna I (TmI) 0,5. Todos metatarsos com tricobótria. Carapaça (Fig. 9) castanho-avermelhada escura com pontilhado negro, glabra, com uma fila de 4-5 cerdas dorsais. Área ocular e fóvea (Figs 9 e 11) enegrecidas. Clípeo alto e glabro. Quelíceras bem desenvolvidas castanho-avermelhadas, com processos espiniformes frontais e laterais (Figs 9-11), cinco dentes na promargem e quatro na retromargem. Esterno (Fig. 10) castanho-escuro com bordas negras. Enditos grandes, castanhoavermelhados. Lábio castanho-escuro. Fila ocular anterior 0,33 e posterior 0,40 . OLP os maiores $(0,10)$, OMA os menores $(0,05)$, OLA e OMP do mesmo tamanho $(0,08)$. Pernas amarelas, exceto coxas com margem posterior pontilhada de negro. Abdome negro-oliváceo. Trocanter do palpo com longo tubérculo pontiagudo (Figs 10 e 11). Êmbolo filiforme, com ápice curvado (Figs 12 e 13), junto à apófise suprategular distal, apófise tibial prolateral alongada e margem do címbio sobre parte do supratégulo (Fig. 13).

Fêmea. Desconhecida.

Distribuição. Sul do Brasil (Paraná).

\section{Labicymbium rusticulum (Keyserling, 1891) Figs $16-20$}

Erigone rusticula Keyserling, 1891: 240-241, fig. 183, holótipo macho, Rio Grande do Sul, Brasil, no BMNH (não examinado); Petrunkevitch, 1911: 237; Bonnet, 1956; 1774.

Erigone rusticola; Mello-Leitão, 1927: 400; Roewer, 1954: 1551. Lapsus calami.

Labicymbium cygnus Ott \& Lise, 1997: 54-56, figs 1-7, holótipo macho, Viamão, Rio Grande do Sul, Brasil, no MCTP (examinado holótipo MCTP e parátipos do MCN); sinonimizada por Miller, 2007: 158; Platnick, 2007.

Diagnose. O palpo do macho apresenta apófise suprategular distal conspícua com ápice redondo (Figs 16-19) mais longa que em L. curitiba sp. nov. (Fig. 12), paracímbio com ápice ventral projetado (Figs 16 e 18). Epígino da fêmea, em vista 

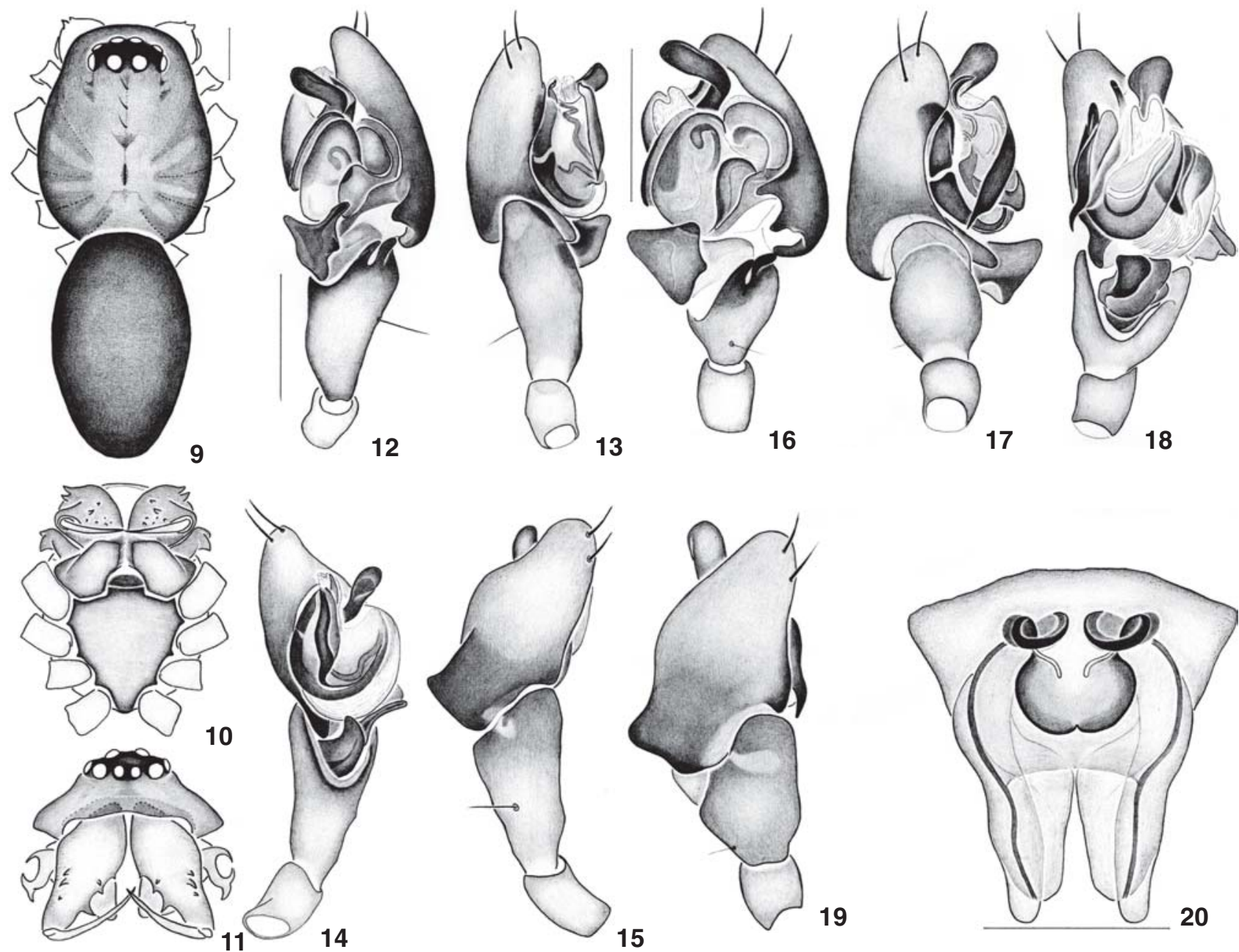

Figuras 9-20. (9-15) Labicymbium curitiba sp. nov.: (9-11) corpo do macho: (9) vista dorsal; (10) vista ventral; (11) vista frontal; (12-15) palpo do macho: (12) vista retrolateral; (13) vista prolateral; (14) vista ventral; (15) vista dorsal; (16-20) L. rusticulum: (16-19) palpo do macho: (16) vista retrolateral; (17) vista prolateral; (18) vista ventral; (19) vista dorsal; (20) epígino, vista dorsal, clarificado. Barras: 0,25 mm.

dorsal clarificada, demonstra que os ductos de copulação são longos e percorrem um trajeto que segue junto aos dois processos na placa epiginal (Fig. 20).

Descrição. Veja OTT \& Lise (1997: 54, 55).

Novos registros. BrasiL, Rio Grande do Sul: Barra do Ribeiro (Fazenda Boa Vista), 1 macho, 16.V.2003, Equipe Probio col. (MCN 35928); Tapes (Fazenda São Miguel), 2 machos, 10 fêmeas, 14.V.2003, Equipe Probio col. (MCN 35935).

Distribuição. Brasil (Rio Grande do Sul).

\section{AGRADECIMENTOS}

Aos curadores das coleções aracnológicas do Museu de Ciências Naturais (MCN) Erica H. Buckup e Museu de Ciência e Tecnologia da PUCRS (MCTP) Arno A. Lise, pelo acesso ao material-tipo. À Erica H. Buckup pelo uso das dependências, bibli- ografias e equipamentos. A direção do $\mathrm{MCN}$ onde este trabalho foi realizado. A Ricardo Ott pelas informações prestadas. Ao CNPq pela concessão da bolsa de doutorado.

\section{LITERATURA CITADA}

Bonnet, P. 1956. Bibliographia araneorum. Analyse méthodique de toute la littérature aranéologique jusqu'en 1939. Toulouse, Les Frères Douladoure, vol. 2, part 2, C-F. 9191926p.

Keyserling, E. 1891. Die Spinnen Amerikas: Brasilianische Spinnen. Nürnberg, Verlag Bauer \& Raspe, 278p.

Mello-Leitão, C.F. 1927. Arachnideos de Santa Catharina (Brasil). Revista do Museu Paulista 15: 393-418.

Miller, J.A. 2007. Review of Erigonine Spider Genera in the Neotropics (Araneae: Linyphiidae, Erigoninae). Zoological

Revista Brasileira de Zoologia 25 (2): 323-327, June, 2008 
Journal of the Linnean Society 149 (Suppl. 1): 1-263. Miluidge, A.F. 1980. The erigonine spiders of North America. Part 1. Introduction and taxonomic background (Araneae: Linyphiidae). The Journal of Arachnology 8: 97-107.

Millidge, A.F. 1991. Further linyphiid spiders (Araneae) from South America. Bulletin of the American Museum of Natural History 205: 1-199.

Otт, R. \& A.A. Lise. 1997. Duas espécies novas de Labicymbium Millidge, 1991 da Região Neotropical (Araneae, Linyphiidae). Biociências 5 (2): 53-59.

Petrunkevitch, A. 1911. A synonymic index-catalogue of spiders of North, Central and South America with all adjacent islands, Greenland, Bermuda, West Indies, Terra del Fuego, Galapagos, etc. Bulletin of the American Museum of Natural History 29: 1-791.

Platnick, N.I. 2007. The world spider catalog, version 8.0. American Museum of Natural History. Disponível em: http:/ /research.amnh.org/entomology/spiders/catalog/index.html [Accessed in 20/IX/2007].

Roewer, C.F. 1954. Katalog der Araneae von 1758 bis 1940. Bruxelles, Institut Royale des Sciences Naturelles, vol. 2b, 925-1751p.

Submitted: 24.X.2007; Accepted: 02.VI.2008.

Editorial responsibility: Geogina Bond-Buckup 